\title{
Contrast Normalization Accounts for Binocular Interactions in Human Striate and Extra-striate Visual Cortex
}

\author{
Chuan Hou, Spero C. Nicholas, and Preeti Verghese \\ Smith-Kettlewell Eye Research Institute, San Francisco, California 94115
}

During binocular viewing, visual inputs from the two eyes interact at the level of visual cortex. Here we studied binocular interactions in human visual cortex, including both sexes, using source-imaged steady-state visual evoked potentials over a wide range of relative contrast between two eyes. The ROIs included areas V1, V3a, hV4, hMT ${ }^{+}$, and lateral occipital cortex. Dichoptic parallel grating stimuli in each eye modulated at distinct temporal frequencies allowed us to quantify spectral components associated with the individual stimuli from monocular inputs (self-terms) and responses due to interaction between the inputs from the two eyes (intermodulation [IM] terms). Data with self-terms revealed an interocular suppression effect, in which the responses to the stimulus in one eye were reduced when a stimulus was presented simultaneously to the other eye. The suppression magnitude varied depending on visual area, and the relative contrast between the two eyes. Suppression was strongest in V1 and V3a (50\% reduction) and was least in lateral occipital cortex (20\% reduction). Data with IM terms revealed another form of binocular interaction, compared with self-terms. IM response was strongest at V1 and was least in hV4. Fits of a family of divisive gain control models to both self- and IM-term responses within each cortical area indicated that both forms of binocular interaction shared a common gain control nonlinearity. However, our model fits revealed different patterns of binocular interaction along the cortical hierarchy, particularly in terms of excitatory and suppressive contributions.

Key words: binocular interaction; dichoptic masking; extra-striate cortex; interocular suppression; normalization; V1

Significance Statement

Using source-imaged steady-state visual evoked potentials and frequency-domain analysis of dichoptic stimuli, we measured two forms of binocular interactions: one is associated with the individual stimuli that represent interocular suppression from each eye, and the other is a direct measure of interocular interaction between inputs from the two eyes. We demonstrated that both forms of binocular interactions share a common gain control mechanism in striate and extra-striate cortex. Furthermore, our model fits revealed different patterns of binocular interaction along the visual cortical hierarchy, particularly in terms of excitatory and suppressive contributions.

\section{Introduction}

Psychophysical studies have documented behavioral responses to dichoptic stimuli with different combinations of target and mask contrasts in the two eyes (Legge, 1984a,b; Maehara and Gpryo, 2005; Ding and Sperling, 2006; Meese et al., 2006). Extensive physiological studies have used dichoptic stimuli to demonstrate interocular suppression in primary visual cortex, V1 (Sengpiel

\footnotetext{
Received Aug. 19, 2019; revised Jan. 10, 2020; accepted Feb. 4, 2020.

Author contributions: C.H. designed research; C.H. performed research; C.H., S.C.N., and P.V. analyzed data; C.H. wrote the first draft of the paper; C.H., S.C.N., and P.V. edited the paper; C.H. wrote the paper; S.C.N. contributed unpublished reagents/analytic tools.

This work was supported by National Institutes of Health Grant R01-EY025018 to C.H. We thank Dr. Jeffrey J. Tsai for sharing and providing the model script used in Tsai et al. (2012); and Margaret Q. McGovern for assistance in recruiting the participants.

The authors declare no competing financial interests.

Correspondence should be addressed to Chuan Hou at chuanhou@ski.org.

https://doi.org/10.1523/JNEUROSCI.2043-19.2020

Copyright $\odot 2020$ the authors
}

and Blakemore, 1994; Sengpiel et al., 1995, 2006; Smith et al., 1997; Truchard et al., 2000; Macknik and Martinez-Conde, 2004; Li et al., 2005; Sengpiel and Vorobyov, 2005; Busse et al., 2009), and in V2 (Bi et al., 2011). The degree of suppression in V1 and V2 was found to be similar in strabismic monkeys (Bi et al., 2011). By contrast, there are few electrophysiological studies of interocular suppression in human V1. Two recent MEG (Chadnova et al., 2017, 2018) and one EEG (Busse et al., 2009) source-imaging studies in humans used a two-frequency dichoptic noisemasking paradigm and varied contrast and luminance levels in the two eyes. They demonstrated interocular suppression in V1, where dichoptic masking decreased responses by $\sim 50 \%$ in participants with normal binocular vision (Chadnova et al., 2018). It is not clear how such binocular contrast interaction propagates from V1 to extra-striate visual cortex.

Our first goal was to obtain a more complete profile of interocular suppression along the human visual cortical hierarchy, including areas of $\mathrm{V} 1, \mathrm{~V} 3 \mathrm{a}, \mathrm{hV} 4, \mathrm{hMT}^{+}$, and lateral occipital cortex 
(LOC). Here we used source-imaged steady-state visual evoked potentials (SSVEPs) with stimuli in each of the two eyes tagged with distinct temporal frequencies (Hou et al., 2016). Our approach was similar to Chadnova et al. $(2017,2018)$, but we used a pair of parallel sinusoidal gratings instead of a noise-masking paradigm. Parallel gratings evoke stronger masking/suppression of the harmonic responses to each eye's stimulus frequency (referred to as self-terms, $\mathrm{nF} 1$ and $\mathrm{nF} 2$ ) than orthogonal gratings (Morrone and Burr, 1986; Burr and Morrone, 1987; Brown et al., 1999; Candy et al., 2001; Moradi and Heeger, 2009). This is true for the neurons in cat's visual cortex as well (Ohzawa and Freeman, 1986; DeAngelis et al., 1992). As self-term responses to each eye's input are reduced by simultaneously presenting stimuli to the other eye (Brown et al., 1999; Chadnova et al., 2017; Chadnova et al., 2018), we investigated dynamic interocular suppression in various cortical areas simultaneously by using different combinations of contrasts in the two eyes.

In addition to self-term responses, the interaction between the two unique frequency components presented to each eye also evokes intermodulation (IM) terms ( $\mathrm{nF} 1 \pm \mathrm{mF} 2)$ (Baitch and Levi, 1988; Suter et al., 1996; Brown et al., 1999; Sutoyo and Srinivasan, 2009; Baker and Wade, 2017; Cunningham et al., 2017). The presence of such IM components constitutes objective neural evidence for interocular interaction (Brown et al., 1999; for review, see Norcia et al., 2015). Therefore, our second goal was to use IM responses as a novel method to measure interocular interaction along the visual cortical hierarchy.

Furthermore, to characterize these two forms of binocular interactions (interocular suppression and interaction revealed by self and IM terms, respectively) and their relation to contrast gain control, we fit both self and IM data simultaneously to a family of divisive gain control models (Tsai et al., 2012) across various cortical areas. Previous masking SSVEP studies (Baker and Wade, 2017; Chadnova et al., 2017, 2018) used a static contrast input in gain control models, which limited their ability to deal with temporal dynamics and the range of input contrasts in our study. The Tsai et al. (2012) model includes a time-varying contrast input and can explain the full range of frequency-domain responses. Model fits of both interocular suppression (self-terms) and interocular interaction (IM terms) provided a more complete description of binocular interactions in human striate and extrastriate visual cortex within the normalization framework of contrast gain control.

\section{Materials and Methods}

Participants

Fifteen participants with normal vision (7 females) between 22 and 68 years old (mean age $44 \pm 14$ years) volunteered for the study. They all had normal or corrected-to-normal vision (20/20 or better in each eye with Bailey-Lovie LogMAR chart). Their stereoacuity was at least 40 arcsec (Random-dot stereo butterfly, Stereo Optical). Their dominant eye and nondominant eye were determined using the hole-in-the card test. The research protocol was approved by the Institutional Review Board of the Smith-Kettlewell Eye Research Institute and conformed to the tenets of the Declaration of Helsinki. Written informed consent was obtained before the experiments.

\section{Experimental design}

Display and stimuli. Figure 1 illustrates the stimuli and experimental design in this study. A pair of 2 cpd parallel sinusoidal gratings was presented on two matched Sony Trinitron monitors (model 110GS) viewed through cross-polarized filters (goggles) at a distance of $100 \mathrm{~cm}$. Each screen had a resolution of $1024 \times 768$ pixels and was refreshed at 85 $\mathrm{Hz}$. The mean luminance of the display was $46.2 \mathrm{~cd} / \mathrm{m}^{2}$. The gratings were contrast-reversed at different temporal frequencies (8.5 and 6.07
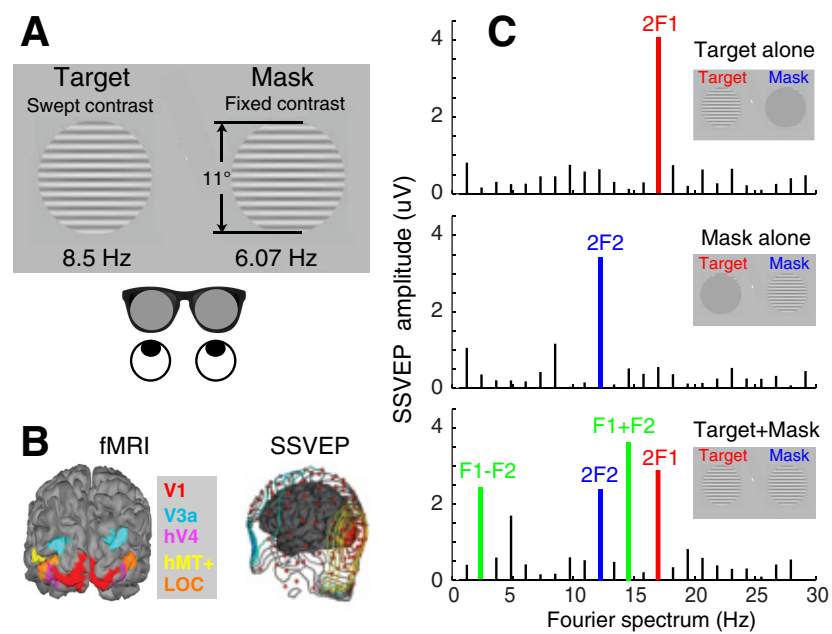

Figure 1. Stimuli and experimental design. $\boldsymbol{A}$, Dichoptic setup for a pair of parallel gratings with distinct temporal frequencies in each eye. $\boldsymbol{B}$, Left, Sample fMRI scan for 1 participant in a separate session to define the ROIs in visual cortical areas. Right, Sample cortical current density from 128 channel EEGs. C, Sample Fourier spectrum and dominant response components in V1 for three experimental conditions. Target alone: the target contrast was swept from $1.7 \%$ to $40 \%$ within a 10 s trial, whereas the mask contrast was set at $0 \%$. Mask alone: the mask contrast was fixed at $20 \%$ for the $10 \mathrm{~s}$ trial, whereas target contrast was set at $0 \%$. Target + Mask: the target contrast was swept from $1.7 \%$ to $40 \%$, whereas the mask contrast was fixed at $20 \%$.

$\mathrm{Hz}$ ) and presented separately to each eye, viewed through cross-polarized filters (as seen in Fig. 1A). Figure $1 B$ shows a sample fMRI scan from 1 participant in a separate session to define the region of interests (ROIs: $\mathrm{V} 1, \mathrm{~V} 3 \mathrm{a}, \mathrm{hV} 4, \mathrm{hMT}^{+}$, and LOC) in visual cortical areas. These ROIs were used for source imaging of EEG scalp potentials. SSVEPs were measured in three conditions from each of 15 participants. In the Target-alone condition, the target grating was contrast-reversed at $8.5 \mathrm{~Hz}$ in the nondominant eye, and its contrast was swept from $1.7 \%$ to $40 \%$ in 10 logarithmic steps within $10 \mathrm{~s}$, while the mask contrast in the dominant eye was set at $0 \%$. The spectrum in this condition was dominated by the second harmonic response (2F1) to the target at $17 \mathrm{~Hz}$ (Fig. $1 C$, top). In the Mask-alone condition, the mask grating was contrast-reversed at 6.07 $\mathrm{Hz}$ in the dominant eye, and its contrast was fixed at $20 \%$ for the trial, which lasted $10 \mathrm{~s}$, while the target contrast in the nondominant eye was set at $0 \%$. The spectrum in this condition was dominated by the second harmonic response (2F2) to the mask at $12 \mathrm{~Hz}$ (Fig. $1 \mathrm{C}$, middle). In the Target + Mask condition, the target contrast in the nondominant eye was swept from $1.7 \%$ to $40 \%$ in 10 -logarithmic steps within $10 \mathrm{~s}$, whereas the mask contrast in the dominant eye was fixed at $20 \%$. The spectrum in this condition consisted of self-terms (Target 2F1 and Mask 2F2) and also IM-terms $(\mathrm{F} 1+\mathrm{F} 2$ at $14.57 \mathrm{~Hz}, \mathrm{~F} 1-\mathrm{F} 2$ at $2.43 \mathrm{~Hz})$, as seen in Figure $1 \mathrm{C}$ (bottom). We also repeated the Target+Mask condition with reversed temporal frequencies in the two eyes, with the target and mask gratings contrast-reversing at 6.07 and $8.5 \mathrm{~Hz}$, respectively. This repeated Target + Mask condition served to determine whether different temporal frequencies affect target and mask responses.

\section{SSVEP data acquisition and source localization}

EEG data were collected from 15 participants with 128-channel HydroCell Sensor Nets and Net Station acquisition system (EGI), bandpass filtered from 0.1 to $50 \mathrm{~Hz}$, and digitized at $500 \mathrm{~Hz}$. Four stimulus conditions (Target alone, Mask alone, Target + Mask, and repeated Target + Mask with reversed temporal frequencies) were presented in a random order with each trial lasting for $10 \mathrm{~s}$ duration that was divided into 12 bins (10 core +1 prelude +1 postlude), and with intervals of $3 \pm 0.5 \mathrm{~s}$ between each trial. The prelude and postlude bins were discarded for data analysis to eliminate onset/offset transients. Twenty trials of each stimulus condition were acquired. Participants were instructed to fixate a central marker and avoid blinking during stimulus presentation. At the end of the EEG session, the 3D locations of 128 sensors and three fiducials (nasion, left and right preauricular) were recorded for each participant 


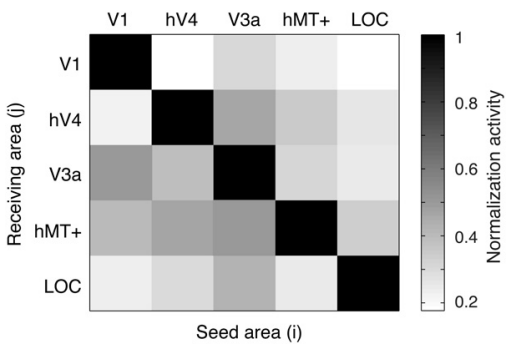

Figure 2. Simulation estimates of cross talk averaged from 15 participants between EEG source-imaged signals in retinotopically defined ROls. The columns show activation in the receiving area (j) when a particular seed area (i) is activated. Activation in the seed areas (diagonal) is much stronger than in other areas (off-diagonal), indicating that the cross talk is modest within visual areas V1, V3a, hV4, hMT ${ }^{+}$, and LOC reported in the study.

using a Fastrak radio-frequency 3D digitizer (Polhemus) and coregistered to participants' T1-weighted anatomical magnetic resonance scans, from which a three-shell boundary element model of the skull and scalp was computed.

EEG signals and artifact rejection (eye movements and blinks) were postprocessed using a custom software package designed by the Norcia research group (Ales et al., 2013). Details for source localization of EEGs are described previously (Appelbaum et al., 2006; Cottereau et al., 2011; Hou et al., 2016; Hou et al., 2017). In brief, an L2 minimum norm inverse was computed with sources constrained to the location and orientation of the cortical surface (Hämäläinen et al., 1993). ROIs corresponding to visual areas V1, V2v, V2d, V3v, V3d, V3a, and hV4 were defined by a separate procedure based on retinotopic mapping using fMRI (Engel et al., 1997). The area $\mathrm{hMT}^{+}$was identified using low-contrast motion stimuli (Huk and Heeger, 2002). The LOC was defined using a blockdesign fMRI localizer scan with stimuli from Kourtzi and Kanwisher (2000).

\section{ROI-based analysis}

In this study, we specifically examined responses in V1, V3a, hV4, $\mathrm{hMT}^{+}$, and LOC. The areas V2 and V3 were excluded due to cross talk from other areas (Cottereau et al., 2011). To measure contrast response functions, raw EEG recordings for each trial were divided into 10 sequential core bins that corresponded to the swept stimulus values (contrast). For each bin, a recursive least-square adaptive filter (Tang and Norcia, 1995) was used to generate a series of complex-valued spectral coefficients representing the amplitude and phase of harmonic responses (Hou et al., 2017). Voltage versus contrast response functions were obtained by coherently averaging the spectral coefficients for each bin across trials for each participant, ROI, harmonic, and stimulus condition. To take into account the different noise levels for each participant (Vialatte et al., 2010), we computed the signal-to-noise ratio (SNR) for each participant by dividing peak amplitudes by the associated noise, which was defined for a given frequency by the average amplitude of the two neighbor frequencies (stimulus frequencies $\pm 1.21 \mathrm{~Hz}$ ). Then, we averaged the SNRs across the 15 participants. As there were no significant differences between left and right hemisphere responses $\left(F_{(1,14)}=3.16\right.$, $p=0.097$ ) when collapsing across stimulus conditions, harmonics and ROIs, we therefore averaged the data from both hemispheres.

\section{Cross talk in ROI}

To estimate the contribution of activities from areas outside the designated ROI, we computed a cross talk matrix, using the calculation described by Lauritzen et al. (2010) and Cottereau et al. (2011), as seen in Figure 2. Cross talk refers to the neural activities generated in different areas that are attributed to a particular ROI due to the smoothing of the electric field by the head volume. Ideally, the cortical current densities would show 0 cross talk, and the associated matrix would be equal to identity; however, the skull, dura, and intervening media smear the source localization. Nevertheless, the visual areas (V1, V3a, hV4, $\mathrm{hMT}^{+}$, and LOC) chosen for our study received on average $<25 \%$ cross talk in our study, allowing us to conclude that the results we observed arise predominantly in the designated areas.

\section{SSVEP contrast threshold estimation}

The SSVEP contrast thresholds are estimated for a given ROI for each individual participant by extrapolating the second harmonic of the SSVEP response amplitude as a function of target contrast to $0 \mathrm{mV}$, using in-house software for contrast sweep VEP (Norcia et al., 1990). The threshold extrapolation algorithm incorporated the signal-to-noise ratio and phase-consistency criteria described by Norcia et al. (1990), with additional cross-checks performed using the $\mathrm{t}^{2}$ circ statistic of Victor and Mast (1991).

\section{Contrast response modeling}

In a nondichoptic masking study, Tsai et al. (2012) extended a wellestablished description of the contrast response function-the hyperbolic ratio function (Naka and Rushton, 1966; Albrecht and Hamilton, 1982) and included a time-varying contrast input that explained the full range of frequency-domain responses. To adapt the Tsai et al. (2012) model to our dichoptic masking paradigm, we made several modifications: (1) we presented the target and mask stimuli to different eyes; (2) we introduced a weighting factor for the mask contrast input as the relative contributions of the target and the mask are different in our dichoptic masking study; and (3) we introduced an additive baseline parameter to account for the SNR floor not being $\sim 1$, as the responses of Target alone $2 \mathrm{~F} 1$ and Target + Mask $2 \mathrm{~F} 2$ were suprathreshold with SNR $>1$. Thus, the variant of the Tsai et al. (2012) model used in our study is described as follows:

$$
\begin{gathered}
c(t)=c_{\text {target }} \frac{\sin \left(2 \pi f_{\text {target }} t\right)+1}{2}+w_{\text {mask }} c_{\text {mask }} \frac{\sin \left(2 \pi f_{\text {mask }} t\right)+1}{2} \\
u(t)=\frac{[c(t)]^{p}}{[c(t)]^{q}+\sigma^{q}} \\
R(f)=R_{0}(f)+R_{m}|U(f)|
\end{gathered}
$$

Equation 1 defines the time-varying contrast $c(t)$, where $c_{\text {target }}$ and $c_{\text {mask }}$ are the contrasts of the Target and Mask, respectively, $f_{\text {target }}$ and $f_{\text {mask }}$ are their temporal frequencies (Carandini, 2004; Bonin et al., 2006; Tsai et al., 2012), and $w_{\text {mask }}$ is a weighting factor of mask contrast relative to target. Equation 2 defines the nonlinearity, where $\sigma$ is a semisaturation constant representing contrast sensitivity, $p$ is an exponent of excitation, and $q$ is an exponent of divisive suppression (Foley, 1994; Chen et al., 2001; Xing and Heeger, 2001; Peirce, 2007), as described by Tsai et al. (2012). Equation 3 is the function fit to the data, where $R(f)$ is the SNR at frequency $f$. The parameter $R_{0}$ is a frequency-dependent baseline parameter we added to account for the signal-to-noise floor, and $R_{\mathrm{m}}$ is the response gain factor. $U(f)$ indicates the amplitude of the Fourier transform of time series $u(t)$ at frequency $f$. Parameter values were obtained by nonlinear constrained optimization (MATLAB function fmincon) to minimize the sum of the squared residual error. The coefficient of determination $\left(R^{2}\right)$ was used to assess goodness of fit of a model. The SDs and CIs of the fit parameter values were estimated from the distributions of 1000 bootstrap resamplings (each of size 15), drawn randomly from participant data with replacement.

\section{Statistical analysis}

Statistical analyses were conducted using repeated-measure ANOVA in R for the data in Figures 3, 4, and 6, and paired $t$ test in Figure 3C.

\section{Results}

\section{Profile of interocular suppression in V1 and}

\section{extra-striate cortex}

Self-term responses represent the response to each eye's input (i.e., target eye and mask eye). As one eye's self-term response is reduced by simultaneously presenting the stimuli to the other eye (Chadnova et al., 2018), we measured this component to inves- 

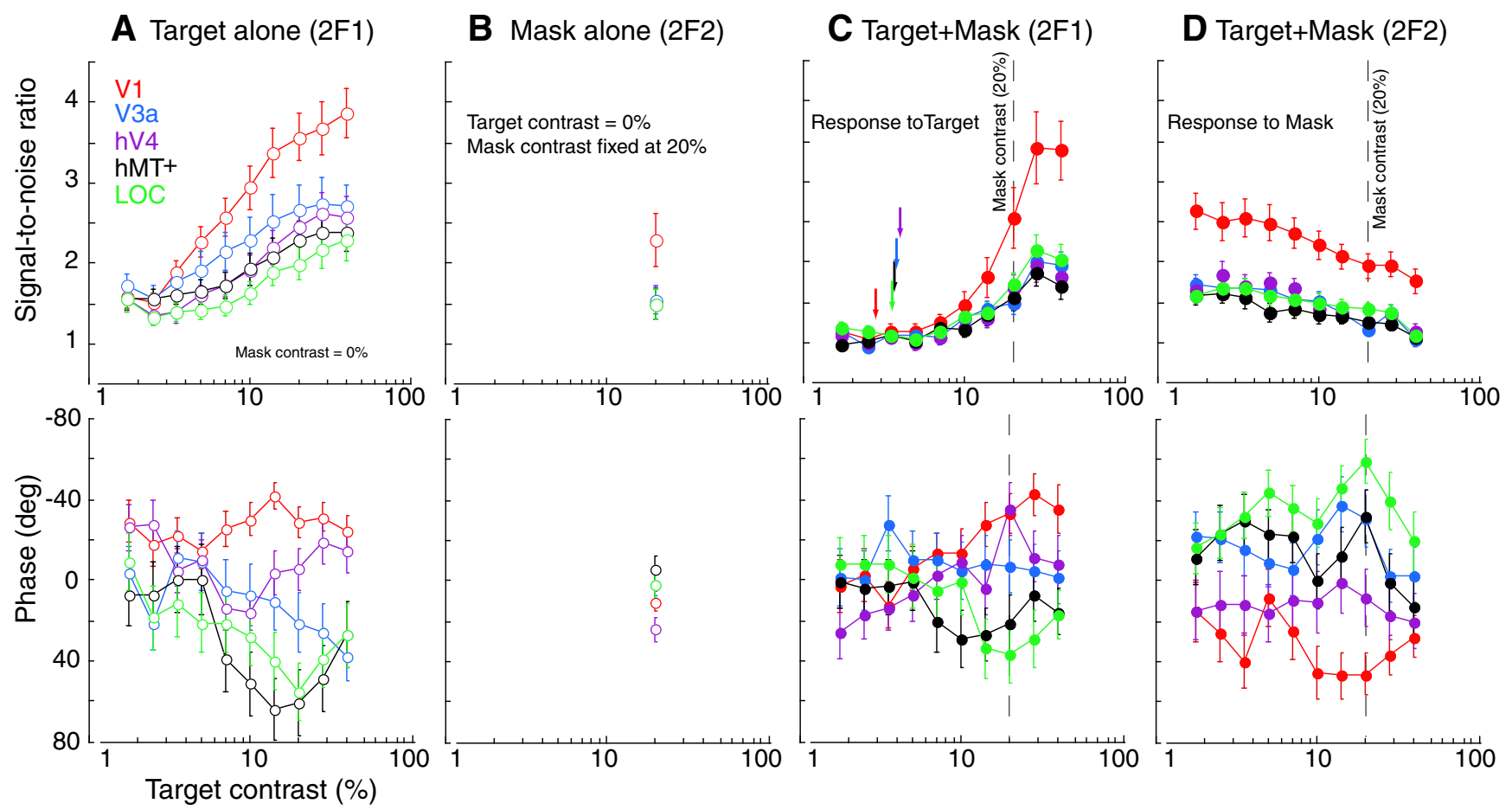

Figure 3. Mean SNR and response phase as a function of target contrast across ROIs. Error bars indicate SEM. Colors represent ROIs. $\boldsymbol{A}$, SNR and phase to the Target-alone stimulus (measured at the frequency $2 \mathrm{~F} 1=17 \mathrm{~Hz}$ ). $\boldsymbol{B}$, SNR and phase to the Mask-alone stimulus (measured at the frequency $2 \mathrm{~F} 2=12.14 \mathrm{~Hz}$ ). SNR and phase to the Target + Mask stimulus, measured at the target frequency $2 \mathrm{~F} 1(\boldsymbol{C})$, and at the mask frequency $2 \mathrm{~F} 2(\boldsymbol{D})$. C, Arrows indicate the averaged individual contrast thresholds measured by extrapolating the SSVEP response amplitude at $2 \mathrm{~F} 1$ versus target contrast response function to $0 \mathrm{mV}$. The response strength (SNR) in V1 was a factor of 2 higher than in extra-striate cortex, regardless of whether the mask was absent or present. Phase changed with increasing target contrast.

tigate interocular suppression under dynamic combinations of contrasts from the two eyes.

Figure 3 plots SNR at the second harmonic responses averaged across 15 participants from 3 stimulus conditions that produced the following self-term response components: the second harmonic of the target frequency when the target was presented alone (Target alone 2F1) and when it was presented with a mask (Target+Mask 2F1), and the second harmonic of the mask frequency when the mask was presented alone (Mask alone 2F2), and when it was presented with the target (Target+Mask 2F2). Data from the different ROIs are shown in different colors. It is obvious that the response was a factor of $\sim 2$ higher in $\mathrm{V} 1$ than in extra-striate cortex, regardless of the absence (Fig. $3 A, B$, top) or presence (Fig. $3 C, D$, top) of a stimulus in the other eye. An initial ANOVA was conducted with the factors of component (Target alone, Target + Mask at 2F1, and Target + Mask at 2F2) and ROI $\left(\mathrm{V} 1, \mathrm{~V} 3 \mathrm{a}, \mathrm{hV} 4, \mathrm{hMT}^{+}\right.$, and LOC) collapsed (averaged) across 10 target contrast levels. The component of Mask alone (Fig. 3B, top) was excluded due to only one contrast level. As expected, the interaction of component and ROI was significant $\left(F_{(8,7)}=5.06\right.$, $p=0.023$ ), suggesting that the responses are different among the conditions and the ROIs. This difference was likely driven by the overall weaker responses in extra-striate cortex, compared with the responses in V1. Therefore, we further compared the responses between $\mathrm{V} 1$ and extra-striate ROIs (averaged responses of areas $\mathrm{V} 3 \mathrm{a}, \mathrm{hV} 4, \mathrm{hMT}^{+}$, and LOC) across 10 target contrast levels for each component. ANOVA with factors of ROI and target contrast level revealed that V1 had significantly stronger responses than extra-striate cortex for all three components: Target alone $2 \mathrm{~F} 1\left(F_{(1,14)}=13.80, p=0.002\right)$, Target + Mask $2 \mathrm{~F} 1\left(F_{(1,14)}\right.$ $=7.97, p=0.014)$, and Target + Mask $2 \mathrm{~F} 2\left(F_{(1,14)}=10.27, p=\right.$
$0.006)$. There were no significant differences among target contrast levels for each component $\left(F_{(9,6)}=2.02, p>0.05\right)$.

Furthermore, as seen in Figure $3 C, D$ when a mask was presented to the other eye, the responses to the Target (Target+ Mask at 2F1; Fig. 3C, top) reduced in all ROIs, compared with Target alone condition (Fig. $3 A$, top). In contrast, the responses to the Mask (Target + Mask at 2F2; Fig. 3D, top) in the presence of a target of increasing contrast decreased monotonically as the target contrast was increased. The pattern of self-term responses at both target frequency (2F1) and mask frequency (2F2) was consistent with the Winner-Take-All competition (Busse et al., 2009; Carandini and Heeger, 2011), as the response seems to be dominated by the higher contrast component. Repeating the Target + Mask condition with reversed temporal frequencies in the two eyes did not show significant differences $\left(F_{(1,14)}=0.32\right.$, $p=0.581$, repeated-measures ANOVA). This result indicated that different temporal frequencies did not affect the pattern of target and mask responses. Therefore, replication of the results suggests good test-retest reliability for the study as well.

We also measured individual contrast thresholds by extrapolating the SSVEP response amplitude at Target + Mask 2F1 versus target contrast response function to $0 \mathrm{mV}$ (Norcia et al., 1990). Previous studies found that extrapolation of contrast response curves efficiently predicts psychophysical thresholds (Campbell and Maffei, 1970). The averaged threshold across participants in each ROI is plotted in Figure 3C (top). Contrast threshold for the target in the presence of a fixed contrast dichoptic mask was $2.9 \%$ in V1, which was significantly lower than the threshold of $3.69 \%$ in extra-striate ROIs (averaged across V3a, hV4, $\mathrm{hMT}^{+}$, and LOC) ( $p=0.011$, paired $t$ test). However, we were not able to measure contrast thresholds for the $2 \mathrm{~F} 1$ component in the target- 

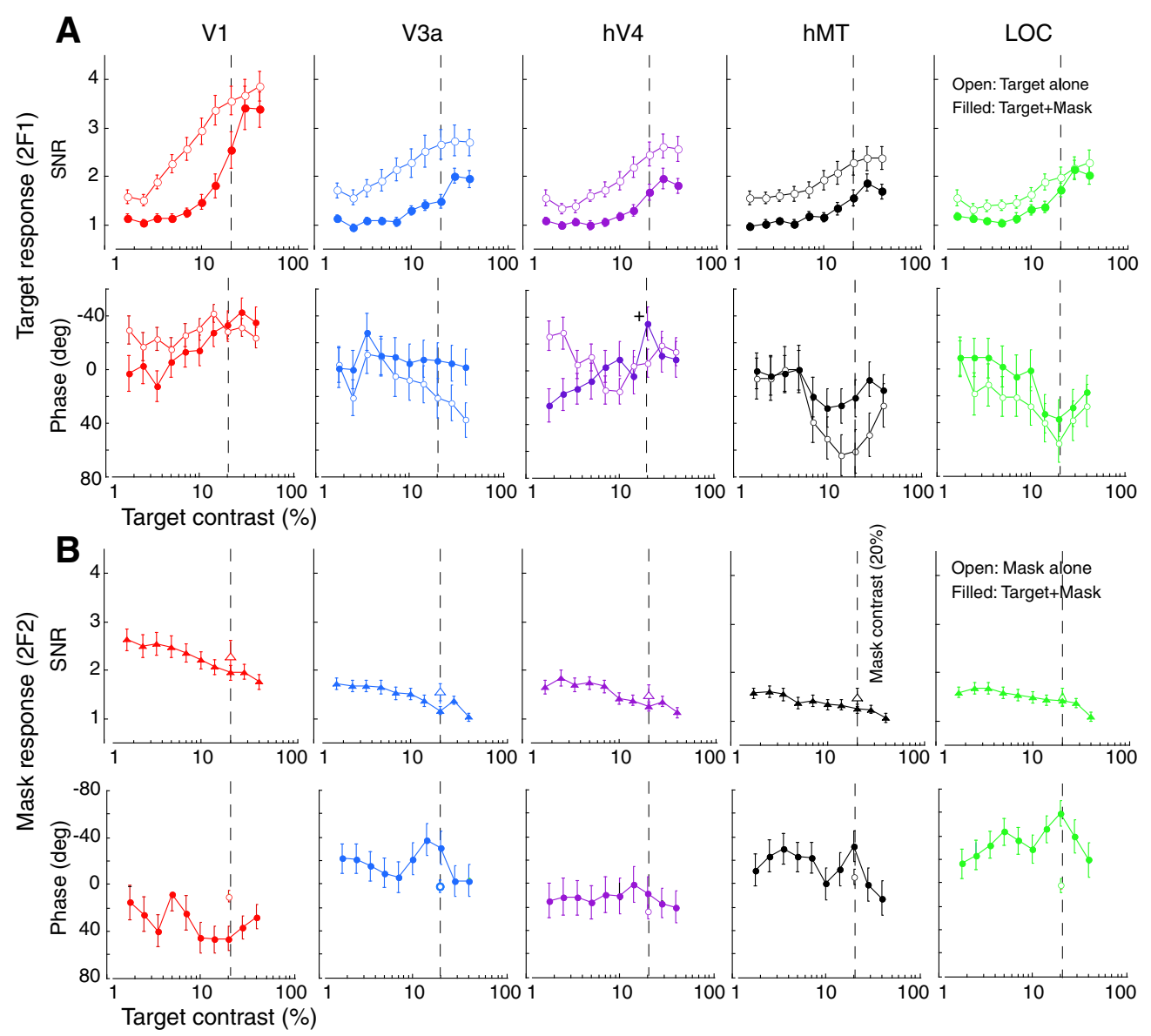

Figure 4. Profile of interocular suppression in various visual cortical ROIs. A, Suppression of the target by the mask. Top, SNR. Bottom, Phase, measured at Target frequency 2F1. B, Suppression of the mask by the target as a function of target contrast. Top, SNR. Bottom, Phase, measured at Mask frequency 2F2. The responses to the target and mask were suppressed by presenting stimuli to the other eye in all ROls. Phase also changed when the mask was present.

alone condition because the contrast sweep started at $1.7 \%$ contrast, which is well above the unmasked threshold measured in a previous study (Norcia et al., 1990). Contrast thresholds measured with a contrast sweep method similar to the one used in our study (Norcia et al., 1990) were $\sim 0.22 \%-0.32 \%$ for adults at low special frequencies $(0.5-2 \mathrm{cpd})$. This should be similar to thresholds for the $2 \mathrm{c} / \mathrm{deg}$ of special frequency grating used in our study. Compared with the thresholds for unmasked stimuli, the thresholds in the presence of a dichoptic mask are approximately an order of magnitude higher (2.9\% in V1 and 3.69\% in extrastriate cortex), suggesting interocular suppression of contrast sensitivity.

The response phase depended on whether the mask was present and whether the responses were to the target or to the mask. In the Target-alone condition, as seen in Figure $3 A$ (bottom), we observed a "phase advance" of the 2F1 component, as described previously (Burr and Morrone, 1987), where the response phase progressively increased with increasing target contrasts, with a steeper increase for intermediate contrasts $(\sim 5 \%-10 \%$ target contrast in our study) in all ROIs, except in V1. V1 showed a phase decrease of $\sim-20^{\circ}$ to $-40^{\circ}$ for intermediate contrasts. When the parallel dichoptic mask was present, the "phase advance" was reduced (from $60^{\circ}$ in Target-alone condition to $40^{\circ}$ in Target+Mask condition) in $\mathrm{hMT}^{+}$and LOC. Interestingly, the "phase advance" in V3a disappeared, and the phase in hV4 showed a "phase decrease" (Fig. 3C, bottom). The phase in V1, as in $\mathrm{hV} 4$, also showed a "phase decrease" with increasing target

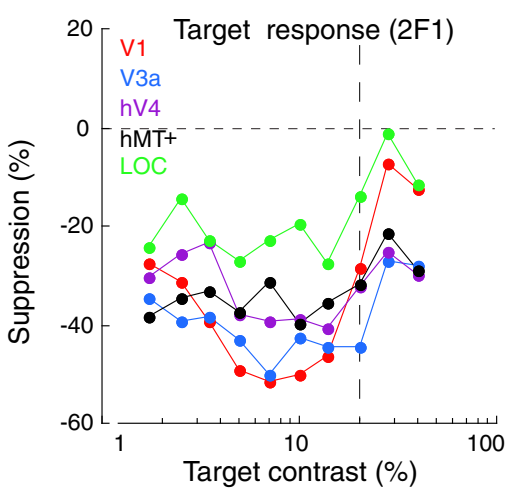

Figure 5. Suppression percentage in various visual cortical ROls. Percentage suppression of the target by the mask, measured at target frequency $2 \mathrm{~F} 1$ as a function of target contrast, while mask frequency was fixed at $20 \%$. Values below and above the horizontal dashed line indicate suppression and facilitation, respectively. Colors represent different ROIs. V1 (red) and V3a (blue) had the strongest suppression ( $\sim 50 \%$ reduction), and LOC (green) had the least suppression ( $\sim 20 \%$ reduction) at $\sim 7 \%$ target contrast.

contrast. These findings suggest that the "phase decrease," including reduction of "phase advance," might be a signature of interocular suppression in parallel dichoptic masking. The observation that the phase advance is abolished in V3a with the introduction of a parallel grating mask is similar to the findings in a previous VEP study (Burr and Morrone, 1987). It is possible that 
the responses recorded at $\mathrm{POz}$ from Burr and Morrone (1987) also represent more dorsal areas, such as V3a.

For the responses to the mask frequency 2F2, the phase showed an opposite pattern, compared with the pattern of the target frequency 2F1 (Fig. 3D, bottom). All ROIs, except V1, showed a "phase decrease" at $\sim 10 \%$ and $20 \%$ target contrast. V1 showed a "phase advance" as described in the Burr and Morrone (1987) study. The opposite pattern of phase change for $2 \mathrm{~F} 1$ and $2 \mathrm{~F} 2$ suggests that the phase responses to the stimuli rely on the relative contrast between the two eyes. A direct comparison of phase responses in each ROI in the Target-alone and Target+ Mask conditions can be seen clearly in the bottom panels of Figure $4 A$ for target frequency (2F1) and of Figure $4 B$ for mask frequency $(2 \mathrm{~F} 2)$.

Figure 4 demonstrates the profile of interocular suppression in V1 and the ROIs in extra-striate cortex. In all ROIs, the responses to the Target (Fig. 4A, top) and to the Mask (Fig. 4B, top) were suppressed (reduced) by presenting stimuli to the other eye. As seen in Figure $4 A$ (top), the effect of the dichoptic mask on the target was to shift all contrast response functions of Target+ Mask 2F1 downward and rightward. The downward and rightward shifts are quantified by the modified version of the Tsai et al. (2012) model, in which Target alone and Target+Mask responses in the top row of Figure $4 A$ were fit separately in each ROI. The detailed fit results are presented below in Monocular versus dichoptic processing in higher visual areas.

Figure 5 plots suppression percentage in each ROI as the difference between with- and without-mask SNR, divided by the Target-alone SNR. Negative and positive values indicate suppression and facilitation, respectively. For suppression of the target by the mask, all ROIs showed suppression from 1.7\% to $40 \%$ target contrast. The suppression was evident at very low target contrasts $(<3.5 \%)$ and reached a maximum at $7 \%$ target contrast, which was far below the contrast at which the two eyes' contrasts were matched (20\%). V1 had the strongest suppression ( $~ 50 \%$ reduction), and LOC had the least suppression ( $\sim 20 \%$ reduction) at the contrast corresponding to peak suppression. The suppression in V3a was similar to V1.

\section{Profile of interocular interaction in V1 and extra-striate cortex}

IM terms can only result from the interaction of the two eyes' inputs; thus, their presence is neural evidence for interocular interaction (Brown et al., 1999). Among the IM components, F1 + F2 was dominant in our study as seen in Figure $1 C$ (bottom), so we used the F1 + F2 IM component to index "interocular interaction" in our study. When both target and mask were present, the IM responses as a function of target contrast across all ROIs were nonmonotonic, as seen in Figure 6 (left): they increased with target contrast to a peak and then declined thereafter. Although overall response magnitudes in extra-striate cortical areas were weaker than in V1, the peak IM responses occurred 20\% target contrast, when the contrast matched that of the mask. In $\mathrm{V} 1$, the IM response became evident at $\sim 2.5 \%$ of target contrast, while in extra-striate ROIs IM response were evident at 4\%-5\% of target contrast. One-factor ANOVA with ROI (V1, V3a, hV4, and $\mathrm{hMT}^{+}$and LOC) at the peak response, where both the target and mask contrast were matched at $20 \%$, revealed significance $\left(F_{(1,14)}=192.24, p<0.001\right)$, suggesting an effect of ROI that is likely driven by higher response in V1. To investigate this further, we compared the responses between V1 and extra-striate ROIs (averaged responses of areas $\mathrm{V} 3 \mathrm{a}, \mathrm{hV} 4, \mathrm{hMT}^{+}$, and $\mathrm{LOC}$ ) across 10 target contrast levels. Similar to the self-terms noted earlier, ANOVA with factors of ROI and target contrast level revealed that V1 had significantly stronger interocular interaction than extra-striate cortex $\left(F_{(1,14)}=7.94, p=0.014\right)$. Among the extrastriate ROIs, hV4 showed the smallest IM response. However, the difference between hV4 and averaged responses of $\mathrm{V} 3 \mathrm{a}, \mathrm{hMT}^{+}$, and LOC did not reach significance $\left(F_{(1,14)}=1.94, p=0.185\right)$. The phase of the IM response also changed with increasing target contrast. However, the pattern of the IM phase change was different from the patterns of phase change in for the 2F1 component in the Target-alone and Target+Mask conditions. V1 and hV4 showed a "phase advance" as contrast increased from $~ 5 \%$ to $\sim 20 \%$ to $40 \%$. The phase in $\mathrm{V} 3 \mathrm{a}, \mathrm{hMT}^{+}$, and LOC showed a small decrease with increasing target contrast and remained at $\sim-40^{\circ}$ for contrasts $>10 \%$.

\section{Monocular versus dichoptic processing in higher visual areas} As mentioned earlier, we quantified the extent of interocular suppression by fitting the target $2 \mathrm{~F} 1$ responses in the absence and presence of mask (Fig. 4A, top row) separately using a divisive gain control model (Tsai et al., 2012) that we modified for our dichoptic masking study. The model fits are shown in Figure 7, where the black solid lines indicate the best fitting model. The corresponding fit parameters and values in each ROI are listed on the left side of the panels for the Target-alone condition and on the right side of the panels for the Target + Mask condition. Several key changes were observed in target $2 \mathrm{~F} 1$ responses in the presence of a dichoptic mask (binocular interaction), compared with those in the absence of mask (monocular processing), which we describe in detail below.

\section{Downward and rightward shifts of the contrast response function in the presence of dichoptic mask}

The changes to the 2F1 response by the addition of a dichoptic mask are characterized primarily by changes to the semisaturation constant $(\sigma)$ and to the baseline parameter $R_{0}$, as shown in Table 1. The $\sigma$ value increased from $4.7 \%$ to $11 \%$ in V1, and from 


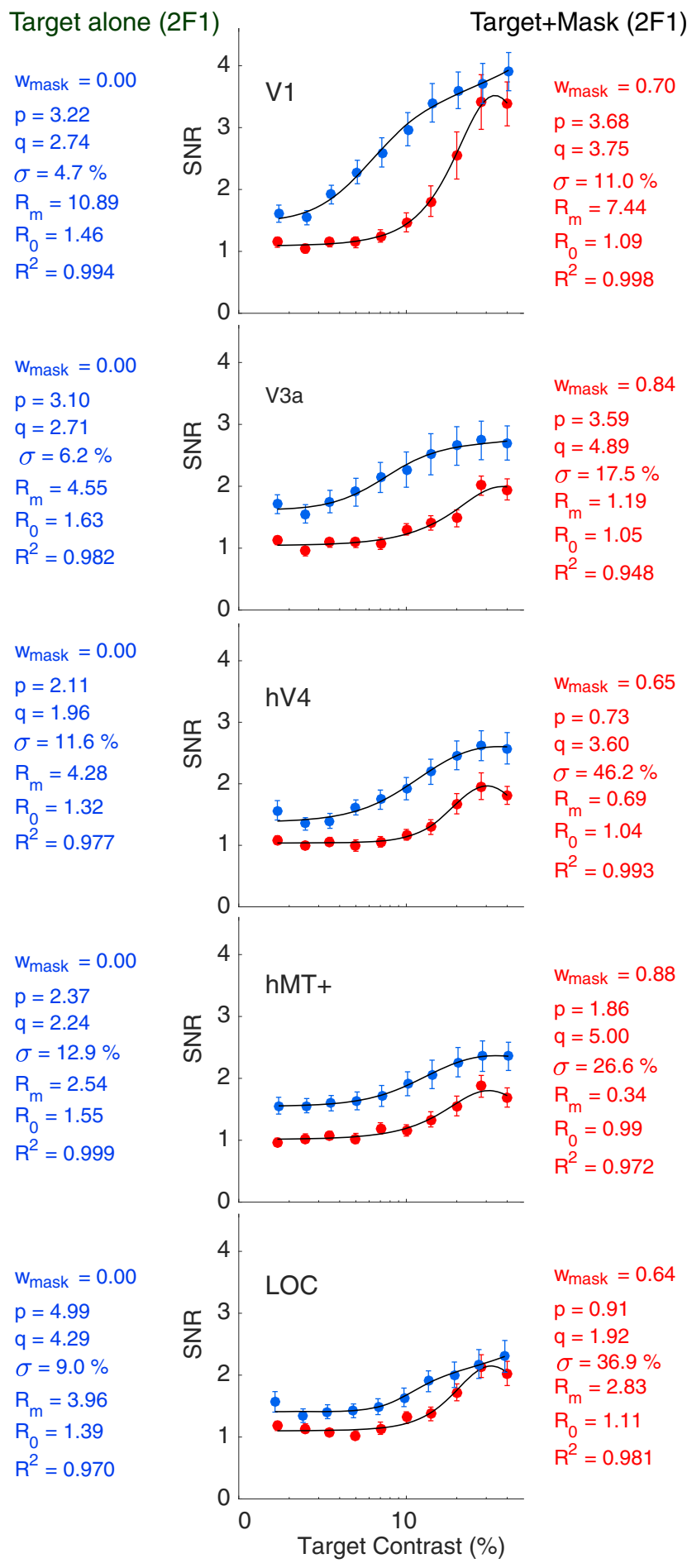

Figure 7. Fits of a variant of the Tsai et al. (2012) model to $2 F 1$ responses in the Target-alone and Target + Mask conditions. Data are replotted from Figure $4 A$ (top). Black solid lines indicate the best fitting model. The corresponding fit parameters in each ROI for the Target-alone and the Target + Mask condition are shown on the left and right, respectively. The fit parameters are as follows: semisaturation constant parameter $(\sigma)$, excitatory $(p)$ and suppressive $(q)$ exponents, response gain factor $\left(R_{\mathrm{m}}\right)$, mask contrast attenuation $\left(W_{\text {mask }}\right)$, and goodness of fit $\left(R^{2}\right)$. $R_{0}$ indicates an additive baseline parameter.

$10 \%$ to $32 \%$ in extra-striate cortex (averaged over extra-striate ROIs). The $\sigma$ values in the presence of mask were distributed over a wide range, showing no significant differences between the ROIs. However, the overall rightward shift of the $2 \mathrm{~F} 1$ responses due to the addition of a dichoptic mask is obvious compared with
Table 1. The $R_{0}$ and $\sigma$ values of fitted parameters from Figure 7

\begin{tabular}{|c|c|c|c|c|c|c|c|c|c|c|}
\hline & \multicolumn{2}{|l|}{ V1 } & \multicolumn{2}{|l|}{ V3a } & \multicolumn{2}{|l|}{ hV4 } & \multicolumn{2}{|l|}{$\mathrm{hMT}^{+}$} & \multicolumn{2}{|l|}{ LOC } \\
\hline & Talone & $T+M$ & Talone & $T+M$ & Talone & $T+M$ & Talone & $T+M$ & T alone & $T+M$ \\
\hline \multicolumn{11}{|l|}{$R_{0}$} \\
\hline Optimal & 1.46 & 1.09 & 1.63 & 1.05 & 1.32 & 1.04 & 1.55 & 0.99 & 1.39 & 1.11 \\
\hline SD & 0.21 & 0.07 & 0.25 & 0.05 & 0.16 & 0.05 & 0.22 & 0.05 & 0.14 & 0.05 \\
\hline \multicolumn{11}{|l|}{$\sigma(\%)$} \\
\hline Optimal & 4.70 & 11.0 & 6.30 & 17.5 & 11.6 & 46.2 & 12.9 & 26.6 & 9.0 & 36.9 \\
\hline SD & 3.76 & 13.3 & 7.14 & 9.99 & 9.65 & 12.2 & 8.95 & 10.9 & 6.48 & 10.9 \\
\hline
\end{tabular}

those in the absence of the mask. The increased $\sigma$ indicates that the mask reduced contrast sensitivity. Table 1 also shows a clear decrease in the baseline level $\left(R_{0}\right)$ on average across ROIs from $1.47 \pm 0.19$ in the absence of the mask to $1.06 \pm 0.05$ in the presence of the mask in all ROIs. The downward shift in the baseline parameter $R_{0}$ is likely due to the fact that even the lowest value of the sweep contrast $(1.7 \%)$ in the Target-alone condition was at a suprathreshold level, which is known to evoke significant VEP responses (Norcia et al., 1990; Hou et al., 2014).

\section{Response gain reduced in the presence of dichoptic mask}

We have seen that the dichoptic mask reduced response amplitudes (Fig. $4 A$, top row). The response gain parameter $R_{\mathrm{m}}$ also showed a similar effect (Fig. 8A). Response gain decreased by a factor of $\sim 2$ in all ROIs in the presence of a dichoptic mask. Response gain in V1 was higher than in extra-striate cortex, regardless of the presence or absence of mask. A previous MEG source-imaging study (Hagler, 2014) also reported that V1 had a factor of $\sim 2$ stronger responses than V2, V3, and V3a for checkerboard pattern stimuli. The strong response gain in V1 might be because the stimuli (contrast in our study, checkerboard in Hagler, 2014) were low level and primarily represented functional properties of V1 neurons. We speculate that the strong response gain in V1 might also be driven by the specific role of V1 as the first locus where binocular information is combined. It is also possible that the more robust EEG responses in V1 are because it has a larger cortical surface area in close proximity to the scalp electrodes, compared with extra-striate cortex, such as areas hV4, $\mathrm{hMT}^{+}$, and LOC.

\section{Excitatory and divisive suppression in monocular and dichoptic processing}

Our model fits revealed a change in the relationship between excitatory $(p)$ and suppressive contributions $(q)$ in the presence of a dichoptic mask. Although the values of $p$ and $q$ differed across cortical ROIs (Fig. 7), divisive suppression across all ROIs was in general stronger than excitation in the presence of a mask, and in general weaker than excitation in the absence of mask. This is evident in Figure $8 B$, where the differences between $q$ and $p$ for the 2F1 component in the Target alone and the Target+Mask conditions are evident. In the absence of mask (monocular processing), $p$ is slightly more dominant (as seen in Fig. $8 B$, bottom, pink). In the presence of mask (dichoptic processing), $q$ is dominant (Fig. $8 B$, top, green) in extrastriate areas. However, this alternation was not observed in V1, where the mean difference between $q$ and $p$ did not reach significance due to the large SD of the fit.

\section{Weight of mask contrast in dichoptic processing}

As seen in Figure 4, overall suppression was weaker from the target eye (swept contrast) to the mask eye (fixed contrast) (Fig. $4 B$, top), compared with the suppression from the mask eye to the 
A

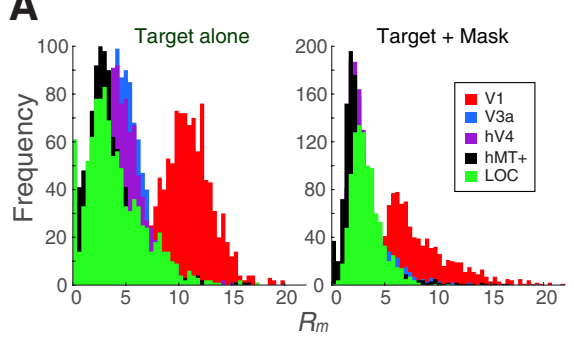

B

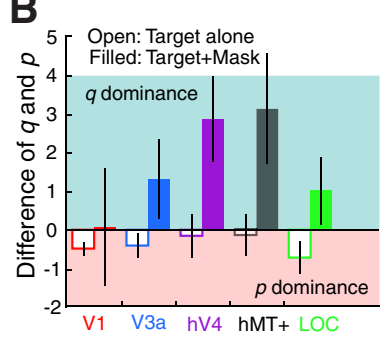

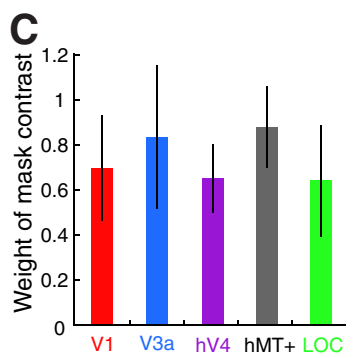

Figure 8. Fit parameters from Figure 7 across ROls. Colors represent the ROls. Error bars indicate the SD from the bootstrap samples. $\boldsymbol{A}$, Histograms of response gain $\left(R_{\mathrm{m}}\right) . R_{\mathrm{m}}$ was lower in the Target + Mask condition, compared with the Target-alone condition; $R_{\mathrm{m}}$ was a factor of 2 higher in $\mathrm{V} 1$ than in extra-striate cortex, regardless of the presence or absence of the mask. $\boldsymbol{B}$, Difference between the suppressive and excitatory exponents ( $q$ and $p$ ) is larger in the presence of mask (filled bars) than in the absence of mask (open bars) in extra-striate cortex, but not in V1. Areas highlighted in green and pink represent divisive suppression $(q)$ and excitatory $(p)$ dominance, respectively. $C$, The values of mask weight $\left(W_{\text {mask }}\right)$ indicate that the mask contrast across R0ls has to be attenuated to $<1$ to achieve good fits of the model.

target eye (Fig. 4A, top). Our model fits are also consistent with this observation. More specifically, we modified the Tsai et al. (2012) model to allow for a parameter that determines the relative contribution of mask contrast, $W_{\text {mask }}$. We found that the effective contrasts of target and mask were different in our dichoptic-masking paradigm, compared with nondichoptic masking (Tsai et al., 2012) where both target and mask contrast inputs had equal weights. This is evident in the values of the weighting factor $\left(W_{\text {mask }}\right)$ in the Target + Mask condition listed in Figure 7 (right) and in Figure $8 C$ (right), where the mask contrast across ROIs had to be attenuated to $\sim 0.74$ on average $( \pm 0.225 \mathrm{SD})$ to achieve good fits of the model. For the dichoptic masking condition, there was no significant difference in $W_{\text {mask }}$ between ROIs. For the Target-alone condition, $W_{\text {mask }}$ was disabled (set to 0 for the fits in Fig. 7 , left). It is not clear why the relative effectiveness of target and mask contrasts were different, but the attenuation of the mask needed to account for the dichoptic masking data suggests that the suppression from the mask eye (fixed contrast) to the target eye (varied contrast) is stronger than the other way around.

\section{Relation of the two forms of binocular interactions to contrast gain control} To characterize the two forms of binocular interaction (i.e., interocular suppression represented by self-terms vs interocular interaction represented by IM terms) and their roles in the relation to contrast gain control in visual cortical hierarchy, we fit all the dichoptic masking data (both self and IM term responses) simultaneously across various cortical areas to a family of divisive gain control models (Tsai et al., 2012) that we modified for our dichoptic masking study. The model fits are shown in Figure 9, where

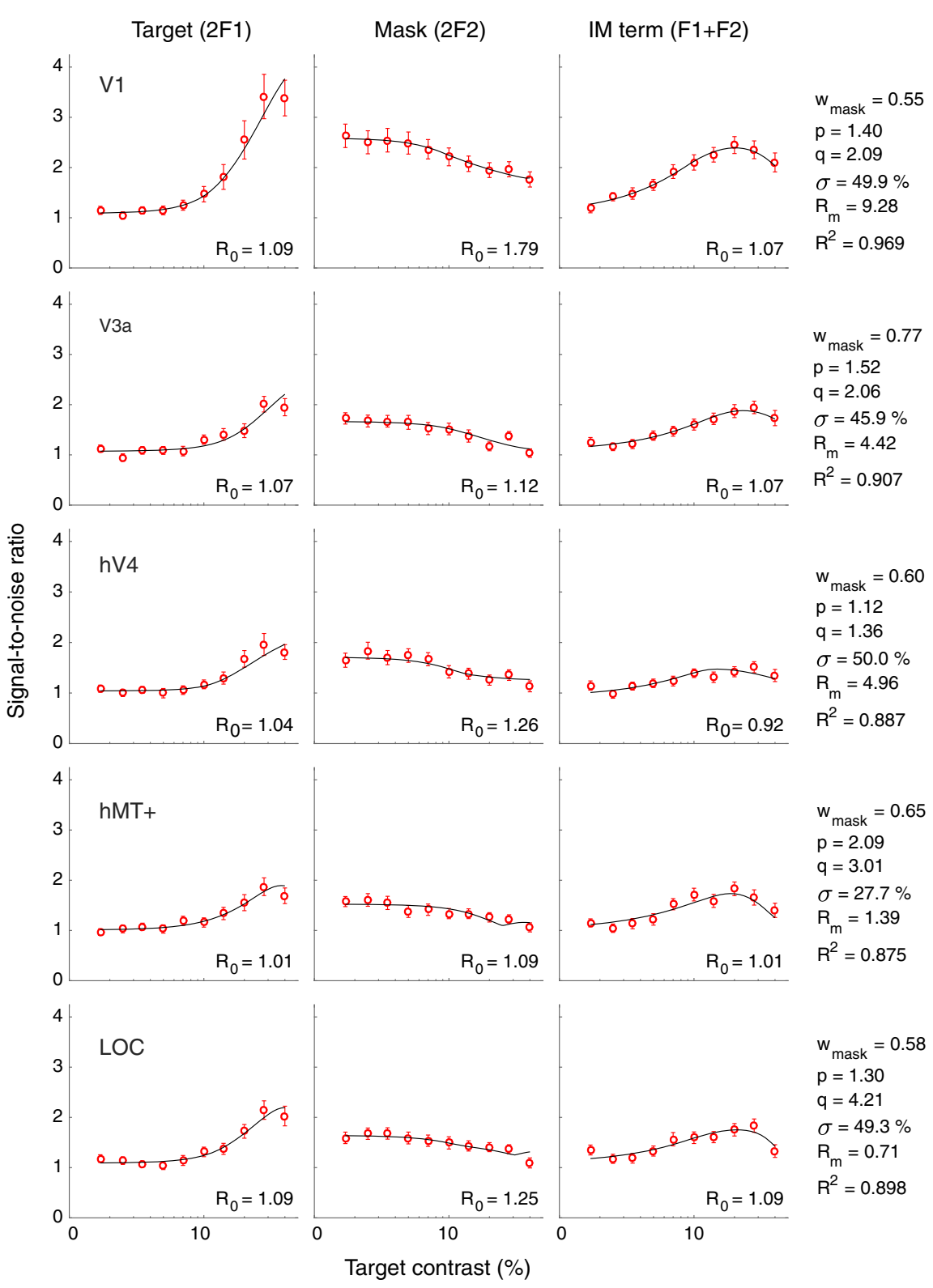

Figure 9. Fits of a variant of the Tsai et al. (2012) model. Data are replotted from Figure $3 C, D$ (top) and Figure $6 A$. Black solid lines indicate the best fitting model. Right, The corresponding fit parameters and values in each ROI. The symbols for fit parameters denote the same meaning described in Figure 7. 
Table 2. Values of fitted parameters from Figure $9^{a}$

\begin{tabular}{llllll}
\hline & V1 & V3a & hV4 & hMT $^{+}$ & LOC \\
\hline$W_{\text {mask }}$ & $0.55 \pm 0.07$ & $0.77 \pm 0.28$ & $0.60 \pm 0.19$ & $0.65 \pm 0.27$ & $0.58 \pm 0.18$ \\
$p$ & $1.40 \pm 1.45$ & $1.52 \pm 1.46$ & $1.12 \pm 1.68$ & $2.09 \pm 1.33$ & $1.30 \pm 1.37$ \\
$q$ & $2.09 \pm 1.11$ & $2.06 \pm 1.37$ & $1.36 \pm 1.57$ & $3.01 \pm 1.28$ & $4.21 \pm 1.31$ \\
$\sigma(\%)$ & $49.9 \pm 17.5$ & $45.9 \pm 17.2$ & $50.0 \pm 18.6$ & $27.7 \pm 13.3$ & $49.3 \pm 14.0$ \\
$R_{\mathrm{m}}$ & $9.28 \pm 4.50$ & $4.42 \pm 3.77$ & $4.96 \pm 2.49$ & $1.39 \pm 1.70$ & $0.71 \pm 2.04$ \\
\hline
\end{tabular}

${ }^{a}$ Values are mean \pm SD.

the black solid lines indicate the best fitting model. The corresponding fit parameters and values in each ROI are listed on the right side of the panels, as well as in Table 2 along with SDs. The fit parameters in Figure 9 typically inherit the patterns of Target+Mask $2 \mathrm{~F} 1$ in Figure 7 , where $W_{\text {mask }}$ is $<1$ and $q$ still remains dominant across all ROIs, as these components (Target 2F1, Mask 2F1, and IM) are all dichoptic responses. However, simultaneously fitting all three response components in a given ROI resulted in larger SDs than fitting a single component (Fig. 7; Target 2F1). Nonetheless, as seen in Figure 9, both self and IM terms in V1 and extra-striate cortex are simultaneously fit well, suggesting that both forms of binocular interactions during dichoptic presentation have the same gain control parameters within a given ROI. The goodness of fit $\left(R^{2}\right)$ varied across the ROIs between 0.875 and 0.967 . This finding indicates that both forms of binocular interactions share a common gain control nonlinearity within a cortical area.

\section{Discussion}

Using source-imaged SSVEP and frequency-domain analysis, we examined the neural dynamics of binocular interactions in various human visual cortical areas over a wide range of relative stimulus contrast between the two eyes. The stimuli in each eye were tagged with distinct temporal frequencies, which allowed us to quantify spectral components associated with the individual stimuli in each eye (self-terms) and the responses due to interaction between the inputs from two eyes (IM terms). These two terms provided a more complete description of neural activities regarding binocular interactions in human striate and extrastriate cortex, including areas $\mathrm{V} 1, \mathrm{~V} 3 \mathrm{a}, \mathrm{hV} 4, \mathrm{hMT}^{+}$, and LOC.

\section{Contrast normalization accounts for two forms of binocular interactions in the visual cortical hierarchy}

One of important findings in this study is that the two forms of binocular interactions (interocular suppression and interaction revealed by self and IM terms, respectively) share a common gain control mechanism. This shared gain control is evident in Figure 9 , in which both self and IM terms are simultaneously fit well not only in V1, but also in extra-striate cortex to a family of divisive gain control models (Tsai et al., 2012). These findings suggest that contrast normalization successfully predicts the neural populations during binocular processing in both striate and extra-striate visual cortex. Our study is the first to demonstrate that interocular interaction as indexed by the IM term is also consistent with divisive gain control in extra-striate cortex.

It is surprising that our dichoptic contrast masking data are fit perfectly well by a contrast gain model developed to explain nondichoptic contrast masking (Tsai et al., 2012), with minor variations, such as presenting target and mask stimuli to different eyes and attenuating mask contrast input. The latter is critical to achieve good fits of the model for dichoptic masking, as it allows for a parameter $\left(W_{\text {mask }}\right)$ that determines the relative contribution of the mask contrast. The original nondichoptic version of the model (Tsai et al., 2012) without $W_{\text {mask }}$ fit our monocular data very well, but not our dichoptic data, as can be seen in Figure $8 \mathrm{C}$. This finding suggests that the relative contributions of each eye to contrast gain may vary under certain circumstances, such as a constant mask in one eye and a varying contrast target in the other eye. We found that the effect from the target eye to the mask eye is weaker than the effect from the mask eye to the target eye. Our variant of the model provides a quantitative estimate of the weighting of contrast from the mask eye $W_{\text {mask }}$, which could be useful to evaluate amblyopic suppression from the nonamblyopic fellow eye to the amblyopic eye.

Previous studies have used a two-stage model (Meese et al., 2006) to describe binocular combination, where the first stage has monocular inputs that are normalized by inputs from both eyes before a second stage where they are combined binocularly. Our data are well fit by a single stage of normalization in Tsai et al. (2012), but that is perhaps because we are measuring responses in $\mathrm{V} 1$ and beyond where the inputs from the two eyes have already been combined binocularly. It is also possible that the relative weighting of the two eyes' input occurs at a first stage, which we implement in our model by attenuating mask contrast before binocular combination. Our dichoptic masking study along with previous dichoptic (Chadnova et al., 2017, 2018) and nondichoptic masking (Candy et al., 2001; Tsai et al., 2012) studies further support the view that normalization serves as a canonical neural computation (Carandini and Heeger, 2011; Baker and Wade, 2017).

\section{Two forms of binocular interactions in the visual cortical hierarchy}

The overall binocular interactions represented by self and IM terms in our study revealed that both forms have stronger responses in V1 than in extra-striate cortex, suggesting that it is likely that the bulk of binocular interactions occurs in V1. This is evident in Figure 7 and its corresponding fit parameters, in which $W_{\text {mask }}$ is similar in all ROIs. In the presence of a dichoptic mask, the decrease in response at low contrast is greatest in V1 and appears to be propagated to higher visual areas (Fig. $3 C$, top). In general, the fit parameters change between V1 and other extrastriate areas; for example, suppression factor $(q)$ becomes dominant in the presence of dichoptic masking only in extra-striate cortex (Fig. 8B). Our model fits suggest that the results are compatible with a gain control model that combines inputs from the two eyes in V1, but allows for cascading stages of gain control in higher visual areas (Simoncelli and Heeger, 1998).

The suppression effect in V1 in our study is similar to the report in human V1 from an MEG source-imaging study (Chadnova et al., 2018), in which dichoptic masking decreased responses by $\sim 50 \%$ in participants with normal binocular vision. Our result in V1 was also similar to low-channel SSVEP responses recorded at $\mathrm{Oz}$ and $\mathrm{POz}$ locations (Baker and Wade, 2017). We should point out that our findings in V1 do not depend critically on SSVEP source imaging, as data from a single electrode at $\mathrm{Oz}$, referenced to the average, are very similar to V1 (Ales et al., 2010). It is possible that the responses at POz in Baker and Wade (2017) represent more dorsal areas, such as $\mathrm{V} 3 \mathrm{a}$, where suppression was found to be similar in magnitude to that found for V1 in our study. An fMRI study reported that V1, V2, and V3 have the same suppression pattern (Moradi and Heeger, 2009). However, we cannot directly compare our data at V2 and V3 with this fMRI study, as there is considerable cross talk from other areas to these two areas in our source-imaging technique (Cottereau et al., 2012, 2015). To our knowledge, there are no comparable studies 
of interocular suppression beyond V3a or higher-level visual cortical areas.

The IM terms revealed another aspect of binocular interaction, compared with self-term responses (interocular suppression) described above and in previous studies (Chadnova et al., 2017, 2018). Our results showed that the IM response was strongest at V1 and was least at hV4. Nevertheless, the peak IM responses occurred at $\sim 20 \%$ target contrast across all ROIs when the target and mask contrasts were equal. IM responses in our study were similar at $\mathrm{V} 3 \mathrm{a}, \mathrm{hMT}^{+}$, and LOC, as these areas are known to be involved in binocular processing and disparity coding (Preston et al., 2008; Cottereau et al., 2011, 2012). There are no comparable studies using IM responses in extra-striate visual cortex.

Although our modeling results suggest that a single contrast gain control mechanism accounts for both the interocular suppression and binocular combination results (self- and IM-term response, respectively) in neurotypical observers, other studies of dichoptic masking in amblyopia suggest that these two processes might be differentially affected in amblyopia. Several studies suggest that interocular suppression is intact (Sengpiel and Blakemore, 1996; Hallum et al., 2017; Shooner et al., 2017), but that binocular combination is compromised (Levi et al., 1979).

\section{Excitatory and suppressive contributions in binocular interactions}

Another important finding in this study is that the contributions from excitation $(p)$ and divisive suppression $(q)$ differed during monocular and dichoptic processing, as seen in Figure 8B. Importantly, our results show, for the first time, that the divisive suppression is stronger in extra-striate cortex than in V1 during dichoptic processing.

\section{Limitation of our model}

We used a variant of the model from Tsai et al. (2012) to explain our dichoptic masking data. As described by Tsai et al. (2012), one limitation of the model is its relative deficiency in predicting the absolute phase of SSVEP responses correctly. Nonetheless, the modeling here for SSVEP response amplitudes does demonstrate that contrast normalization accounts for binocular interactions in human striate and extra-striate visual cortex.

In conclusion, we studied neural dynamics of binocular interactions at different levels of human visual cortex over a wide range of relative contrast between two eyes. We measured two forms of binocular interactions: one associated with the individual stimuli that represent interocular suppression from each eye, and the other a direct measure of interocular interaction between inputs from the two eyes. We demonstrated that these two forms of binocular interactions share a common gain control mechanism in both striate and extra-striate cortex but have different characteristics in areas along the visual cortical hierarchy. Furthermore, our model fits revealed two important characteristics of dichoptic masking: attenuation of mask contrast and dominance of divisive suppression in extra-striate cortex. Our study provides a more complete description of binocular interaction in human striate and extra-striate cortex and provides the basis for comparison with clinical populations with abnormal binocular vision, such as strabismus and amblyopia in future studies.

\section{References}

Albrecht DG, Hamilton DB (1982) Striate cortex of monkey and cat: contrast response function. J Neurophysiol 48:217-237.

Ales JM, Yates JL, Norcia AM (2010) V1 is not uniquely identified by polar- ity reversals of responses to upper and lower visual field stimuli. Neuroimage 52:1401-1409.

Ales JM, Appelbaum LG, Cottereau BR, Norcia AM (2013) The time course of shape discrimination in the human brain. Neuroimage 67:77-88.

Appelbaum LG, Wade AR, Vildavski VY, Pettet MW, Norcia AM (2006) Cue-invariant networks for figure and background processing in human visual cortex. J Neurosci 26:11695-11708.

Baitch LW, Levi DM (1988) Evidence for nonlinear binocular interactions in human visual cortex. Vision Res 28:1139-1143.

Baker DH, Wade AR (2017) Evidence for an optimal algorithm underlying signal combination in human visual cortex. Cereb Cortex 27:254-264.

Bi H, Zhang B, Tao X, Harwerth RS, Smith EL 3rd, Chino YM (2011) Neuronal responses in visual area V2 (V2) of macaque monkeys with strabismic amblyopia. Cereb Cortex 21:2033-2045.

Bonin V, Mante V, Carandini M (2006) The statistical computation underlying contrast gain control. J Neurosci 26:6346-6353.

Brown RJ, Candy TR, Norcia AM (1999) Development of rivalry and dichoptic masking in human infants. Invest Ophthalmol Vis Sci 40: $3324-3333$.

Burr DC, Morrone MC (1987) Inhibitory interactions in the human vision system revealed in pattern-evoked potentials. J Physiol 389:1-21.

Busse L, Wade AR, Carandini M (2009) Representation of concurrent stimuli by population activity in visual cortex. Neuron 64:931-942.

Campbell FW, Maffei L (1970) Electrophysiological evidence for the existence of orientation and size detectors in the human visual system. J Physiol 207:635-652.

Candy TR, Skoczenski AM, Norcia AM (2001) Normalization models applied to orientation masking in the human infant. J Neurosci 21:45304541.

Carandini M (2004) Receptive fields and suppressive fields in the early visual system, Ed 3. Cambridge, MA: Massachusetts Institute of Technology.

Carandini M, Heeger DJ (2011) Normalization as a canonical neural computation. Nat Rev Neurosci 13:51-62.

Chadnova E, Reynaud A, Clavagnier S, Hess RF (2017) Latent binocular function in amblyopia. Vision Res 140:73-80.

Chadnova E, Reynaud A, Clavagnier S, Baker DH, Baillet S, Hess RF (2018) Interocular interaction of contrast and luminance signals in human primary visual cortex. Neuroimage 167:23-30.

Chen CC, Kasamatsu T, Polat U, Norcia AM (2001) Contrast response characteristics of long-range lateral interactions in cat striate cortex. Neuroreport 12:655-661.

Cottereau BR, McKee SP, Ales JM, Norcia AM (2011) Disparity-tuned population responses from human visual cortex. J Neurosci 31:954-965.

Cottereau BR, McKee SP, Ales JM, Norcia AM (2012) Disparity-specific spatial interactions: evidence from EEG source imaging. J Neurosci 32: $826-840$.

Cottereau BR, Ales JM, Norcia AM (2015) How to use fMRI functional localizers to improve EEG/MEG source estimation. J Neurosci Methods 250:64-73.

Cunningham DG, Baker DH, Peirce JW (2017) Measuring nonlinear signal combination using EEG. J Vis 17:10.

DeAngelis GC, Robson JG, Ohzawa I, Freeman RD (1992) Organization of suppression in receptive fields of neurons in cat visual cortex. J Neurophysiol 68:144-163.

Ding J, Sperling G (2006) A gain-control theory of binocular combination. Proc Natl Acad Sci U S A 103:1141-1146.

Engel SA, Glover GH, Wandell BA (1997) Retinotopic organization in human visual cortex and the spatial precision of functional MRI. Cereb Cortex 7:181-192.

Foley JM (1994) Human luminance pattern-vision mechanisms: masking experiments require a new model. J Opt Soc Am A Opt Image Sci Vis 11:1710-1719.

Hämäläinen M, Hari R, Ilmoniemi R, Knuutila J, Lounasmaa OV (1993) Magnetoencephalography: theory, instrumentation and applications to the non invasive study of human brain function. Rev Mod Phys 65:413497.

Hagler DJ Jr (2014) Visual field asymmetries in visual evoked responses. J Vis $14: 13$.

Hallum LE, Shooner C, Kumbhani RD, Kelly JG, García-Marín V, Majaj NJ, Movshon JA, Kiorpes L (2017) Altered balance of receptive field excita- 
tion and suppression in visual cortex of amblyopic macaque monkeys. J Neurosci 37:8216-8226.

Hou C, Norcia AM, Madan A, Good WV (2014) Visuocortical function in infants with a history of neonatal jaundice. Invest Ophthalmol Vis Sci 55:6443-6449.

Hou C, Kim YJ, Lai XJ, Verghese P (2016) Degraded attentional modulation of cortical neural populations in strabismic amblyopia. J Vis 16:16.

Hou C, Kim YJ, Verghese P (2017) Cortical sources of Vernier acuity in the human visual system: an EEG-source imaging study. J Vis 17:2.

Huk AC, Heeger DJ (2002) Pattern-motion responses in human visual cortex. Nat Neurosci 5:72-75.

Kourtzi Z, Kanwisher N (2000) Cortical regions involved in perceiving object shape. J Neurosci 20:3310-3318.

Lauritzen TZ, Ales JM, Wade AR (2010) The effects of visuospatial attention measured across visual cortex using source-imaged, steady-state EEG. J Vis 10:pii:39.

Legge GE (1984a) Binocular contrast summation: I. Detection and discrimination. Vision Res 24:373-383.

Legge GE (1984b) Binocular contrast summation: II. Quadratic summation. Vision Res 24:385-394.

Levi DM, Harwerth RS, Smith EL 3rd (1979) Humans deprived of normal binocular vision have binocular interactions tuned to size and orientation. Science 206:852-854.

Li B, Peterson MR, Thompson JK, Duong T, Freeman RD (2005) Crossorientation suppression: monoptic and dichoptic mechanisms are different. J Neurophysiol 94:1645-1650.

Macknik SL, Martinez-Conde S (2004) Dichoptic visual masking reveals that early binocular neurons exhibit weak interocular suppression: implications for binocular vision and visual awareness. J Cogn Neurosci 16:1049-1059.

Maehara G, Gpryo K (2005) Binocular, monocular and dichoptic pattern masking. Opt Rev 12:76-82.

Meese TS, Georgeson MA, Baker DH (2006) Binocular contrast vision at and above threshold. J Vis 6:1224-1243.

Moradi F, Heeger DJ (2009) Inter-ocular contrast normalization in human visual cortex. J Vis 13.1-22.

Morrone MC, Burr DC (1986) Evidence for the existence and development of visual inhibition in humans. Nature 321:235-237.

Naka KI, Rushton WA (1966) S-potentials from luminosity units in the retina of fish (Cyprinidae). J Physiol 185:587-599.

Norcia AM, Appelbaum LG, Ales JM, Cottereau BR, Rossion B (2015) The steady-state visual evoked potential in vision research: A review. J Vis 15:4.

Norcia AM, Tyler CW, Hamer RD (1990) Development of contrast sensitivity in the human infant. Vision Res 30:1475-1486.

Ohzawa I, Freeman RD (1986) The binocular organization of complex cells in the cat's visual cortex. J Neurophysiol 56:243-259.
Peirce JW (2007) The potential importance of saturating and supersaturating contrast response functions in visual cortex. J Vis 7:13.

Preston TJ, Li S, Kourtzi Z, Welchman AE (2008) Multivoxel pattern selectivity for perceptually relevant binocular disparities in the human brain. J Neurosci 28:11315-11327.

Sengpiel F, Blakemore C (1994) Interocular control of neuronal responsiveness in cat visual cortex. Nature 368:847-850.

Sengpiel F, Blakemore C (1996) The neural basis of suppression and amblyopia in strabismus. Eye (Lond) 10:250-258.

Sengpiel F, Vorobyov V (2005) Intracortical origins of interocular suppression in the visual cortex. J Neurosci 25:6394-6400.

Sengpiel F, Freeman TC, Blakemore C (1995) Interocular suppression in cat striate cortex is not orientation selective. Neuroreport 6:2235-2239.

Sengpiel F, Jirmann KU, Vorobyov V, Eysel UT (2006) Strabismic suppression is mediated by inhibitory interactions in the primary visual cortex. Cereb Cortex 16:1750-1758.

Shooner C, Hallum LE, Kumbhani RD, García-Marín V, Kelly JG, Majaj NJ, Movshon JA, Kiorpes L (2017) Asymmetric dichoptic masking in visual cortex of amblyopic macaque monkeys. J Neurosci 37:8734-8741.

Simoncelli EP, Heeger DJ (1998) A model of neuronal responses in visual area MT. Vision Res 38:743-761.

Smith EL 3rd, Chino Y, Ni J, Cheng H (1997) Binocular combination of contrast signals by striate cortical neurons in the monkey. J Neurophysiol 78:366-382.

Suter S, Suter PS, Perrier DT, Parker KL, Fox JA, Roessler JS (1996) Differentiation of VEP intermodulation and second harmonic components by dichoptic, monocular, and binocular stimulation. Vis Neurosci 13: $1157-1166$.

Sutoyo D, Srinivasan R (2009) Nonlinear SSVEP responses are sensitive to the perceptual binding of visual hemifields during conventional 'eye' rivalry and interocular 'percept' rivalry. Brain Res 1251:245-255.

Tang Y, Norcia AM (1995) An adaptive filter for steady-state evoked responses. Electroencephalogr Clin Neurophysiol 96:268-277.

Truchard AM, Ohzawa I, Freeman RD (2000) Contrast gain control in the visual cortex: monocular versus binocular mechanisms. J Neurosci 20:3017-3032.

Tsai JJ, Wade AR, Norcia AM (2012) Dynamics of normalization underlying masking in human visual cortex. J Neurosci 32:2783-2789.

Vialatte FB, Maurice M, Dauwels J, Cichocki A (2010) Steady-state visually evoked potentials: focus on essential paradigms and future perspectives. Prog Neurobiol 90:418-438.

Victor JD, Mast J (1991) A new statistic for steady-state evoked potentials. Electroencephalogr Clin Neurophysiol 78:378-88.

Xing J, Heeger DJ (2001) Measurement and modeling of center-surround suppression and enhancement. Vision Res 41:571-583. 